\title{
With thanks to our 2020 peer reviewers
}

We are grateful to the following individuals for their significant contribution to Health Promotion and Chronic Disease Prevention in Canada as peer reviewers in 2020. Their expertise ensures the quality of our journal and promotes the sharing of new knowledge among peers in Canada and internationally.

Amira Ali
Laura Anderson
Maulik Baxi
Suzanne Beno
Caroline Bergeron
Michael Borghese
Danielle Bouchard
Jennifer Ann Brown
Leslie Carlin
Catherine Carstairs
Helen Cerigo
Martin Chartier
Jaime Cidro
Rosa Darling
Louise de Lannoy
Andrea Deierlein
Melissa deJonge

Rose Anne Devlin

Minh T. Do

Melissa J. DuPont-Reyes

Aganeta Enns

Geneviève Gariépy

Allison Gates

Naomi Gibbs

Anton Käll

Jodi Kalubi

Boris Kralj

Claudia Lagacé

Karen Le

Alison Macpherson

Kerry McBrien

Angela Meadows

Katrina Milaney

Luke Mondor

\author{
Howard Morrison \\ Jessica Murphy \\ Jennifer O’Loughlin \\ Adeolu Oladunjoye \\ Scott B. Patten \\ Louise Pilote \\ Stephanie Prince Ware \\ Mark Raizenne \\ Javier Sevil-Serrano \\ Helen Slater \\ Hannah Thompson \\ Joslyn Trowbridge \\ Claire Tugault-Lafleur \\ Nithya Vijayakumar \\ Irene Vikman \\ Michelle Vine
}

